\title{
Nursing approach to the use of buttonhole and rope ladder cannulation of arteriovenous fistula based on a survey in Nigeria
}

\author{
$\mathrm{MJARED}^{1 *}$ and $\mathrm{V} \mathrm{RAJKI}^{2}$ \\ ${ }^{1}$ Diaverum Dialysis Centre, Budapest, Hungary \\ ${ }^{2}$ Department of Nursing, Faculty of Health Sciences, Semmelweis University, Budapest, Hungary
}

(Received: December 21, 2017; revised manuscript received: June 3, 2019; accepted: September 3, 2019)

\begin{abstract}
Purpose: The main purpose of this study is to examine the nursing aspect of two types of cannulation techniques and to investigate the outcomes of using cannulations on an arteriovenous fistula, as well as analysing their impact on outcome. Materials and methods: Data were collected using a self-developed questionnaire. Data were obtained from haemodialysis (HD) patients in Rivon Dialysis Centre in the city of Rivers State named Port Harcourt with over $60 \mathrm{HD}$ patients and with over $15 \mathrm{HD}$ nurses. Only 50 patients met the inclusive criteria after answering the questionnaire, 25 for rope ladder (RL) and 25 for buttonhole (BH) and 10 nurses chosen answered the questionnaire. Results: This study revealed that patients who are using the BH cannulation technique reported a better outcome than the RL group patients. Patients reported lower occurrence of complication, for instance, out of 25 patients, 8 of them using the BH and 15 of them using the RL reported to have infection. Conclusion: Based on the results, it is possible to conclude that the patients who are cannulated with the RL cannulation technique have a higher risk of experiencing complications than the patients using the $\mathrm{BH}$ site cannulation technique.
\end{abstract}

Keywords: buttonhole cannulation techniques, rope ladder cannulation techniques, haemodialysis, arteriovenous fistula, nursing

\section{INTRODUCTION}

Evidently, end stage renal failure (ESRF) has become a serious health care problem affecting the population. Furthermore, patients with end stage renal disease (ESRD) under haemodialysis (HD) rely on functioning vascular access (VA) to enable effective regular treatment. Therefore, cannulation is an important issue for nurses as well.

The native arteriovenous fistula (AVF) was the most commonly used method in adult patients with ESRF due to the lower risk of complications [1]. After AVF has been surgically created, matured for a few weeks, and made ready for cannulation prior to treatment, the fistula could be used for HD using the buttonhole cannulation $(\mathrm{BH})$ technique (also known as constant-site or same-site) or the rope ladder cannulation (RL) technique (also known as site-rotation or rotating sites). The former one was called area cannulation technique, but it not recommended because of its high-risk complication [2]. Figure 1 demonstrates the differences between the certain techniques.

The well-known $R L$ cannulation technique involves cannulating the entire length of the fistula at a different site every time to allow healing of the previous puncture site. This technique might be problematic for patients with a short fistula length or patients whose fistula is difficult to cannulate [5]. There is an alternative method called the constant-site, commonly known as the $B H$ site cannulation technique. In the $\mathrm{BH}$ method, the same nurse creates a permanent hole and inserts the needle to the exact same spot with the same angle of cannulation. After the hole is well formed, the blunt needle can be switched for the following HD sessions [5]. Twardowski [6] was the first physician to report his experience using the $\mathrm{BH}$ site cannulation technique in the United States medical literature. He found it to be a better cannulation option for patients with a native fistula [5]. The RL cannulation technique is vital for the preservation of the AVF and complication risk reduction, since complications may result in loss of VA [7].

\section{International studies on the BH cannulation technique compared to the RL cannulation technique}

Several publications have appeared in the past years documenting the experiences with $\mathrm{BH}$ and LR cannulation techniques and much research on this theme has been conducted. The previous research studies on cannulation techniques are focused on the RL and $\mathrm{BH}$ techniques, in which BH is recommended by the National Kidney Foundation's Kidney Disease Outcome Quality Initiative (NKF/ KDOQI). The outcomes of primary patency, episodes of bacteraemia, access blood flow, and quality of life scores between RL and $\mathrm{BH}$ patients were compared. In an article,

* Corresponding author: Jared Merit; Diaverum Dialysis Centre, H-1118 Dayka Gábor utca 30, Budapest, Hungary; Phone: +36 20 503 5659; E-mail: meritjared@gmail.com

This is an open-access article distributed under the terms of the Creative Commons Attribution-NonCommercial 4.0 International License, which permits unrestricted use, distribution, and reproduction in any medium for non-commercial purposes, provided the original author and source are credited, a link to the CC License is provided, and changes - if any - are indicated. 

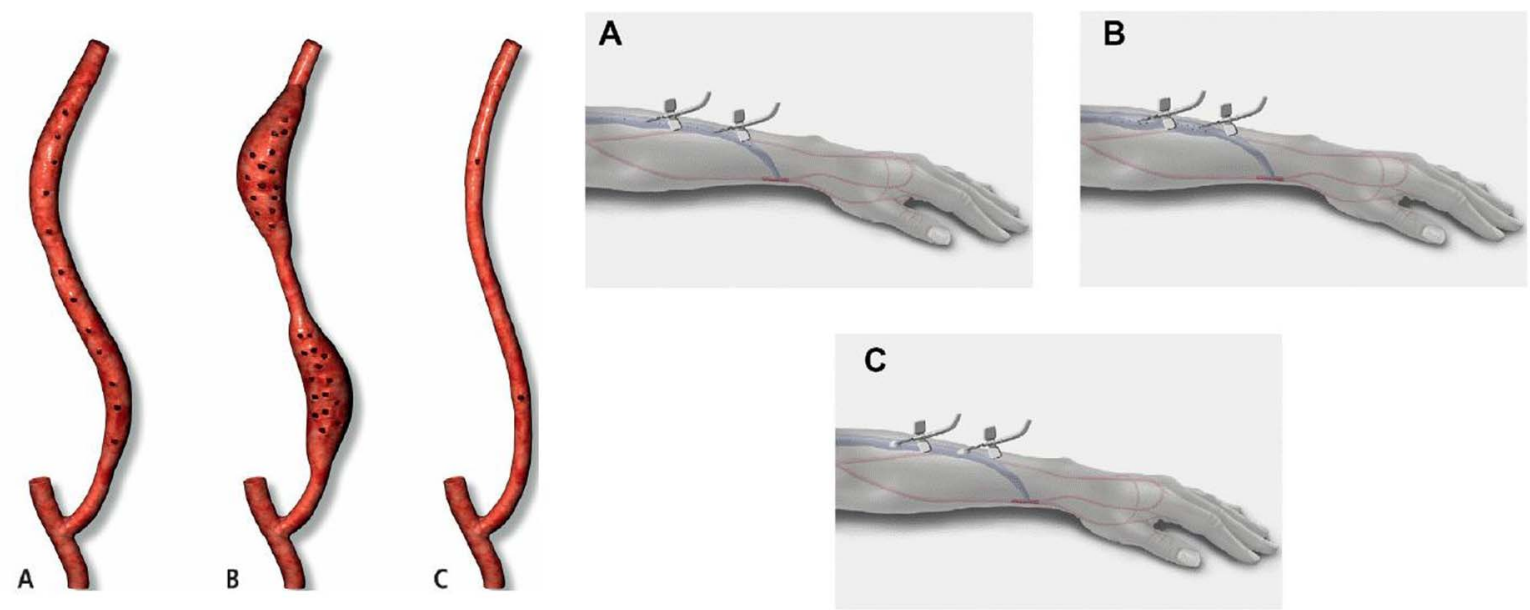

Figure 1. Cannulation techniques - (A) RL technique, (B) area technique, and (C) BH technique $[3,4]$

the authors performed a descriptive and exploratory study in Brazil to determine the benefits of using the $\mathrm{BH}$ cannulation technique in AVF. Fifteen patients were interviewed under HD in the city of Rio de Janeiro in May 2014. They focused on pain, well-being, aesthetics, quality of life, and safety. The BH technique has contributed satisfactorily and appreciably to the quality of life of patients. The authors associated this technique with low levels of pain; safety and quality, as well as the preservation of aesthetics and body image; and the sense of well-being was found [5, 8-15].

\section{Nursing interventions before cannulation of $V A$}

Assessment of the VA. Assessment of VA is fundamentally based on inspection, palpation, and auscultation. The condition of the VA has to be compared and contrasted with the findings on previous assessments. The assessment must be carried out at every cannulation. As for inspection, nurses must observe the general development of the AVF, skin condition, aneurysms including false aneurysms, the skin colour, warmth, and dryness of extremities; check for swellings, signs of infection, and capillary refill $(<2-3 \mathrm{~s})$; and see if there are any ischaemic spots on the fingertips. Furthermore, the following conditions must be ruled out: infection, central or outflow vein stenosis, and steal syndrome. Infection is marked by redness, drainage, and abscess; vein can be diagnosed based on skin colour, oedema, and small purple veins; while in steal syndrome, the hands appear to be cold and painful, and the fingers go numb. As for auscultation, the vascular sounds of the access must be evaluated: the quality and amplitude of the bruit must be assessed; any unusual pitch changes, like whistle sounds, must be detected as they may indicate stenosis; and it must be checked whether the systolic and diastolic sounds are louder on the arterial side. As for palpation, thrills and pulsations must be felt by the nurse. Normally, a thrill is present at the anastomosis site, which disappears with manual occlusion of the AVF, unless accessory veins have been formed. This thrill should decrease in the venous limb of the AVF. If any other thrill is felt outside of the anastomosis, it can be an indication of stenosis. Furthermore, the vein diameter must be palpated by checking the length of the AVF, selecting potential needle sites, looking for flat spots, and deciding whether a new AVF can be cannulated $[2,16]$.

Skin preparation before cannulation for both $B H$ and $R L$. First of all, patients must be instructed to wash their arms with $2 \%$ chlorhexidine at the hand basin, before sitting in the dialysis seat. Second, before the fistula cannulation starts, the nurse must also cleanse the patient's arm with $2 \%$ chlorhexidine in $70 \%$ alcohol. Moreover, for $\mathrm{BH}$ patients, a cotton ball absorbed with chlorhexidine/alcohol $70 \%$ must be used to disinfect the BHs for softening before scab removal. Finally, the scabs must be removed with an $18 \mathrm{G}$ dull needle and followed by another site cleansing with chlorhexidine/alcohol $70 \%$ prior to administering local anaesthetics if used and/or introducing the fistula needle [11].

\section{MATERIALS AND METHODS}

\section{Assessments/measurements}

The main objective of this research work is to determine the outcomes of using the $\mathrm{BH}$ site cannulation technique compared to the RL cannulation in reducing complication haematoma formation, aneurysm development, infection, bleeding, stenosis, and thrombosis among HD patients in Nigeria.

This research work is also aimed at providing the necessary knowledge to help HD nurses to improve on their needling/cannulation skills. We used a new approach, because only a few or no research studies have been carried out on this theme in Nigeria, and this research will provide an insight into this topic.

This study will also carry out research to assess the pain levels of patients using the $\mathrm{BH}$ or RL cannulation. In addition, the satisfaction level/confidence level of HD patients using the $\mathrm{BH}$ compared to the RL cannulation is also investigated. Finally, an assessment of the satisfaction level/confidence level of HD nurses in Nigeria using the $\mathrm{BH}$ compared to the $\mathrm{RL}$ cannulation technique is carried out. 


\section{Participants}

A cross-sectional study was conducted to examine the outcomes of using these two cannulation techniques. In 2017, this study was carried out on ESRD-diagnosed patients under HD through AVF in Nigeria, who started their first cannulation prior to HD treatment 3-10 months before our survey, and their fistula was also created at the same time. Ten HD nurses were also included in this survey. The patients were divided into two groups, the first group consisted of 30 patients using the BH cannulation technique and the second group was a group of 30 patients using the RL cannulation technique. All the patients were 18 or $>18$ years. Only 50 patients met the inclusive criteria after answering the questionnaire, 25 for $\mathrm{RL}$ and 25 for $\mathrm{BH}$.

Data were obtained from current ESRD patients under $\mathrm{HD}$ in the southern region of Nigeria, Rivon Dialysis Center in the city of Rivers State named Port Harcourt with over 60 HD patients and with over $10 \mathrm{HD}$ nurses.

\section{Procedures}

We developed a self-made questionnaire for the patients, and the patients were assisted by the staff during completion of the questionnaire.

The self-made questionnaire was divided into six sections for HD patients and four sections for HD nurses: (a) demographic characteristics of patients/nurses, (b) type of cannulation technique in both patients and nurses, (c) complications patients have had using the cannulation technique (haematoma, bleeding, aneurysm, bleeding, stenosis, thrombosis, and infection), (d) pain level of the patients, (e) satisfaction level, and (f) confidence level of both patients and nurses. All the items were used to answer the objectives of the study.

\section{Statistical analyses}

Data analysis was carried out using the 2010 Excel Microsoft Program. Most of the calculations were carried out manually. Comparison of complications between $\mathrm{BH}$ and $\mathrm{RL}$ technique was performed using $\chi^{2}$ test. Frequencies were used to describe categorical variables. Mean and standard deviations were used to describe pain level, satisfaction level, and confidence level. Statistical significance level was set at $p=.05$.

\section{Inclusion and exclusion criteria}

In this research, the patients have used AVF or newly created AVF at least 3-6 months old, 7-9 months old, and $>10$ months ago. They were at least 18 years old and above. They were mentally fit to be able to read and understand the developed questionnaire and they were also examined by the nephrologists to ensure that they are capable of using the $\mathrm{BH}$ or RL technique.

Patients who were not mentally fit in answering, who were less than 18 years of age, and who used the arteriovenous graft and central venous catheter for HD were also all excluded from this research.

\section{Pilot study}

Upon receiving ethical approval from "Rivon Clinic" nephrologists and ward nurses, the pretesting of the questionnaire was undertaken on 10 registered adult patients under HD. Patients were randomly chosen to assess the feasibility and simplicity of the self-developed questionnaire, prior to the main proposed research work. These patients were not included in the main research work. Out of 10 patients who participated in the pilot study, 4 used the $\mathrm{BH}$ cannulation technique, whereas 6 used the RL cannulation technique. Their response rate was excellent, and the results obtained from $\mathrm{BH} / \mathrm{RL}$ patients were the same. There were no differences between the two techniques from the pilot study result. Since the response rate was excellent with the self-developed questionnaire, we decided to carry out the main research work using the same questionnaire with a larger sample size to determine the differences between these two cannulation techniques.

\section{RESULTS}

\section{HD patients' results}

Out of the 60 questionnaires that were sent out, only 50 of the questionnaires were answered (the total sample size of patients was 50): 25 for $\mathrm{BH}$ and RL cannulation techniques, respectively. Ten of the questionnaires were not completed.

$\mathrm{BH}$ cannulation technique was used in the cases of 25 patients and RL cannulation technique was used for 25 patients as well.

Description of demographic characteristics and statistical analysis for complications of the total population. Table 1 summarizes the demographic characteristics of the patients: sex, AVF age, and time of first cannulation.

Table 2 summarizes the total number of patients who have or have not had complications using the cannulation techniques.

Pain-level score for $B H / R L$ cannulation technique. Patients who were using the $\mathrm{BH} / \mathrm{RL}$ cannulation technique reported pain levels as shown in Table 3 during establishment and after establishment of the cannulation site. The mean value and standard deviation are included in the table.

Total satisfaction-level and total confidence-level mean scores for both techniques - Patients. For satisfaction level, patients using the $\mathrm{BH}$ cannulation reported a total mean value of $2.67 \pm 1.04$, whereas patients using the RL technique reported a mean value of $3.09 \pm 1.29$. For confidence level, patients using the $\mathrm{BH}$ cannulation had a total mean value of $3.41 \pm 1.06$. Patients using the RL technique had a total mean value of $2.86 \pm 1.12$ (Figure 2).

\section{HD nurses' results}

Six out of $10 \mathrm{HD}$ nurses who answered the questionnaire stated they preferred using the RL cannulation technique subjectively, whereas the rest (i.e., 4 nurses) stated they prefered the $\mathrm{BH}$ site cannulation technique. 
Table 1. Demographic characteristics of study population

\begin{tabular}{lcccc}
\hline & Group & BH $(n=25)$ & RL $(n=25)$ & Total $(N=50)$ \\
\hline Sex & Male & 11 & 8 & 19 \\
Fistula age & Female & 14 & 17 & 31 \\
& $3-6$ months old & 9 & 9 & 18 \\
First cannulation & $7-9$ months old & 8 & 7 & 15 \\
& $>10$ months old & 8 & 9 & 17 \\
& 3-6 months ago & 13 & 7 & 20 \\
& $7-9$ months ago & 7 & 11 & 18 \\
\hline
\end{tabular}

Note. BH: buttonhole cannulation technique; RH: rope ladder cannulation technique.

Table 2. Data reported by patients whether or not they had complications using BH/RL cannulation technique

\begin{tabular}{|c|c|c|c|c|c|}
\hline & Group & $\mathrm{BH}(n=25)$ & $\mathrm{RL}(n=25)$ & Total $(N=50)$ & $p$ value \\
\hline \multirow[t]{2}{*}{ Infection } & Yes & 8 & 15 & 23 & .047 \\
\hline & No & 17 & 10 & 27 & \\
\hline \multirow[t]{2}{*}{ Aneurysm development } & Yes & 9 & 17 & 26 & .024 \\
\hline & No & 16 & 8 & 24 & \\
\hline \multirow[t]{2}{*}{ Haematoma formation } & Yes & 15 & 17 & 32 & .556 \\
\hline & No & 10 & 8 & 18 & \\
\hline \multirow[t]{2}{*}{ Bleeding } & Yes & 10 & 14 & 24 & .258 \\
\hline & No & 15 & 11 & 26 & \\
\hline \multirow[t]{2}{*}{ Stenosis } & Yes & 10 & 14 & 24 & .258 \\
\hline & No & 15 & 11 & 26 & \\
\hline \multirow[t]{2}{*}{ Thrombosis } & Yes & 10 & 12 & 22 & .395 \\
\hline & No & 15 & 13 & 28 & \\
\hline
\end{tabular}

Note. Bold values represent statistically significant values in $\chi^{2}$ test. BH: buttonhole cannulation technique; RH: rope ladder cannulation technique.

Table 3. Mean for level of pain

\begin{tabular}{lcc}
\hline Pain-level scale $(1-5)^{\mathrm{a}}$ & $\begin{array}{c}\mathrm{BH} \\
(\text { mean } \pm S D)\end{array}$ & $\begin{array}{c}\mathrm{RL} \\
(\text { mean } \pm S D)\end{array}$ \\
\hline $\begin{array}{c}\text { During establishment of } \\
\text { cannulation site }\end{array}$ & $3.48 \pm 0.87$ & $3.76 \pm 1.16$ \\
$\begin{array}{c}\text { After establishment of } \\
\text { cannulation site }\end{array}$ & $2.44 \pm 1.04$ & $3.84 \pm 1.28$ \\
\hline
\end{tabular}

Note. $\mathrm{BH}$ : buttonhole cannulation technique; $\mathrm{RH}$ : rope ladder cannulation technique; $S D$ : standard deviation.

aPain-level scale: 1 - no pain, 2 - mild pain, 3 - moderate pain, 4 - painful, 5 - extremely painful.

Total satisfaction-level and total confidence-level mean scores for both techniques - HD nurses. For satisfaction level, nurses who preferred using the $\mathrm{BH}$ cannulation had a total mean value of $3.58 \pm 0.93$, whereas the nurses that prefer the RL technique had a mean value of $2.50 \pm 0.81$.

For confidence level, nurses who preferred using the $\mathrm{BH}$ cannulation had a total mean value of $3.92 \pm 0.72$, whereas the nurses who preferred the RL technique had a total mean value of $2.44 \pm 0.56$ (Figure 3 ).

\section{LIMITATIONS OF THIS STUDY}

Although the overall findings of this study help us to understand the outcomes of using two different types of cannulation techniques on an AVF, it is important to

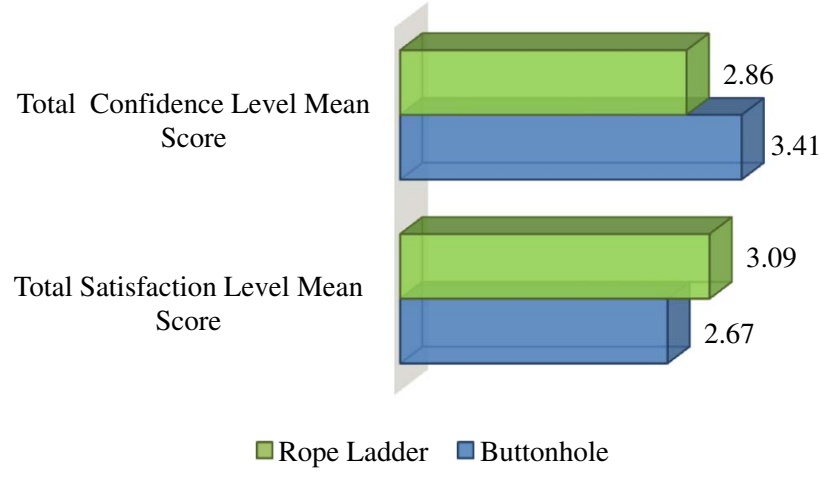

Figure 2. Comparison of patients' satisfaction-level total mean score with confidence-level mean score for BH/RL (satisfaction-

level scale: 1-5: 1 - strongly agree, 5 - strongly disagree; confidence-level scale: 1-5: 1 - extremely confident,

$$
5 \text { - not at all confident) }
$$

acknowledge some limitations of the study. The main limitation of the experimental results is the fact that it is a cross-sectional study on a small number of patients $(N=50)$ and in a single HD centre in Nigeria, and only a small population out of all the patients participated in this study and therefore the study findings cannot be generalized and conclusive. Certainly, it would be of better value if it was conducted in a multicentre design on a larger number of patients. Some of the patients refused to answer the questionnaire because ESRD in Nigeria has always been referred to by the Nigerian nephrologists as a death 


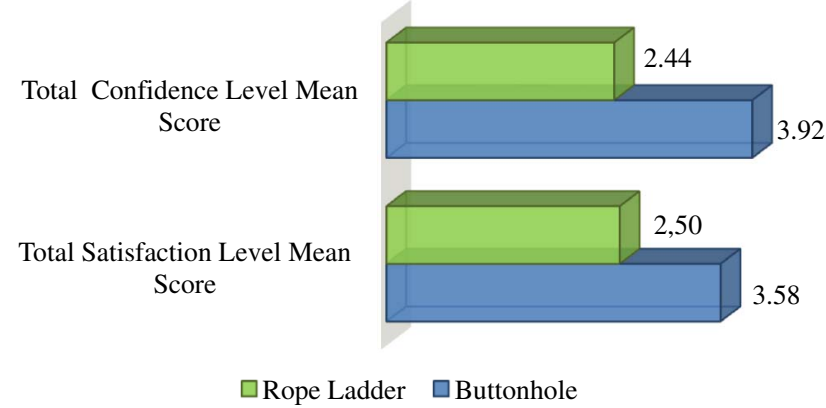

Figure 3. Comparison of HD nurses' satisfaction-level total mean with confidence-level total mean score for using BH/RL technique (satisfaction-level scale: 1-5: 1 - strongly agree, 5 - strongly disagree; confidence-level scale: 1-5:

1 - extremely confident, 5 - not at all confident)

sentence, so they reported that answering the questionnaire will make them feel very sad and will remind them of their limited time to be in this world.

In the light of limitations, further study of the issue would still be required with a larger sample from diverse regions of the world and also in various cities in Nigeria.

\section{THE STRENGTHS OF THIS STUDY}

The response rate was excellent and all items of the questionnaires were answered by all 50 participants. We developed the questionnaire, which is understandable for the participants. Similarly, we received excellent responses from the $10 \mathrm{HD}$ nurses who participated, and they answered honestly and without the use of resources/references related to this study. Finally, this is the first study about the cannulation techniques used on an AVF in Rivon Clinic. Therefore, this will enable the HD nurses in Nigeria to know the kind of cannulation technique to be used on an AVF safely, with respect to precautionary measures.

\section{DISCUSSION}

This is one of the few studies that approach the outomes of using $\mathrm{BH}$ and $\mathrm{RL}$ cannulation techniques in an AVF.

In this study, the results show that both cannulation techniques cause complications, but patients using the $\mathrm{BH}$ cannulation had low occurrence for complications compared to patients using the RL technique. Out of the total number of the patients using the $\mathrm{BH}$ site cannulation technique, 8 reported they had infection, 9 had aneurysm, 15 had haematoma, 10 had bleeding, 10 had stenosis, and 10 of them reported having thrombosis. Meanwhile, out of the total number of the patients using the RL cannulation technique, 15 of them reported they had infection, 17 had aneurysm, 17 had haematoma, 14 bleeding, 14 had stenosis, and 12 of them reported having thrombosis. These results have revealed that patients using the RL technique reported higher number of complications due to the technique than the BH group patients. This study found that the differences between infection and aneurysm formation between the groups are significant $(p=.047$ and $p=.024)$.
This result is similar to some other studies. For instance, a prospective observational study [8] also comparing the $\mathrm{BH}$ and RL techniques of AVF cannulation found that $\mathrm{BH}$ technique had notable benefits. Their result showed that patients who were using RL and switched to $\mathrm{BH}$ while having existing aneurysms that had developed with the RL technique also showed a tendency to flatten out while using the $\mathrm{BH}$ technique. No aneurysms occurred with the $\mathrm{BH}$ technique, few patients from the $\mathrm{BH}$ group developed infection and thrombosis. Similarly, Smyth et al. [11] conducted a prospective cohort study in 2013 among 104 patients on consistent HD via AVF. These authors found that there was higher infection and haematoma occurrence for the RL group compared to the $\mathrm{BH}$ group, but it was not statistically significant. Occurrence of aneurysm was higher in the RL group compared to the $\mathrm{BH}$ group and was statistically significant.

Furthermore, an international cross-sectional survey of VA cannulation practices obtained similar results when investigating 171 dialysis units in the following countries: Portugal, United Kingdom, Ireland, Italy, Turkey, Romania, Slovenia, Poland, and Spain. Originally, 10,807 patients were enrolled in the survey, and access survival data were available for 7,058 patients from these countries. About $90.6 \%$ of the participants had an AVF. Area technique was used in $65.8 \%$, RL technique in $28.2 \%$, and $\mathrm{BH}$ technique in $6 \%$ as access needling. Kaplan-Meier curves were calculated, and multivariable Cox regression model was applied. The most important result for our topic was that the "area cannulation was associated with a significantly higher risk of access failure than RL or BH' [12].

In the "Pain level of patients using BH/RL techniques" part of this study, the pain-level score was assessed during and after establishment of cannulation site on a scale of 1-5 to know which of the cannulation techniques has high pain level experienced by patients. Patients using BH/RL technique had almost the same level of pain during establishment of cannulation site with a mean score of $3.48 \pm 0.87$ and $3.76 \pm 1.16$ for $\mathrm{BH} / \mathrm{RL}$ technique, respectively. A greater proportion of patients in the RL group had excess pain, which was defined by a mean of $3.84 \pm 1.16$ after establishment of cannulation site compared to the $\mathrm{BH}$ group with a mean score of $2.44 \pm 1.04$ after establishment of cannulation site. The differences between the pain score for after establishment of cannulation site were very wide apart, which indicates that patients using the RL technique experience higher pain during receiving haemodiaysis treatment.

Wong et al. [13] conducted a systematic review of randomized trials and observational studies on cannulation pain. The observational studies yielded a statistical reduction in pain with $\mathrm{BH}$ cannulation, but no difference in cannulation pain was found among randomized controlled trials.

Two previous research studies $[14,15]$ on cannulation techniques are focused on RL and $\mathrm{BH}$ cannulation techniques, respectively. BH cannulation is recommended by the $\mathrm{NKF} / \mathrm{KDOQI}$. The outcomes of primary patency, episodes of bacteraemia, access blood flow, and quality of life scores between RL and $\mathrm{BH}$ patients were compared by Chan et al. [14]. The authors used a prospectively collected VA database. 
Forty-five prevalent dialysis patients using $\mathrm{BH}$ were compared with 38 patients using the RL technique over a median of 12 months (interquartile range: 4-27 months). There was no significant difference in demographics of the two groups, except for diabetes mellitus, which was typical in those using $\mathrm{BH}$ as compared to RL. As a result, risk factors associated with lack of primary patency were: female gender and age. In this research, the use of the $\mathrm{BH}$ technique was not associated with improved primary patency. There was no significant difference in the episodes of bacteraemia and mean scores from KDQOL36 between the groups. $\mathrm{BH}$ use is not associated with improved access patency, which was first proved by this study [14]. Da Silver et al. [15] also found that there is reduction in pain level for $\mathrm{BH}$ compared to the $\mathrm{RL}$ technique.

In the "Satisfaction/confidence level for HD patients using BH/RL techniques" part of this study, various questions were asked to rate the satisfaction level. $\mathrm{BH}$ group patients reported higher level of satisfaction than the RL group, which was rated after calculating the total mean score for satisfaction level for each group. BH group patients reported a higher level of satisfaction using this technique with a total mean score of $2.67 \pm 1.04$ than the RL group patients with a total mean of $3.09 \pm 1.29$, which signifies that they are not so satisfied using the technique. Some similar studies have been carried out to know the satisfaction level of HD patients using these techniques.

In the observational study conducted by Hashmi et al. [5] on patients who were using the RL technique and then switched to the $\mathrm{BH}$ technique investigated the patients' experience and attitude in comparison to the previous technique. Then, they were reassessed 12 months later and they found that $96 \%$ said they would recommend the $\mathrm{BH}$ technique. High level of satisfaction using the $\mathrm{BH}$ site cannulation was found, with a high positive attitude in suggesting the $\mathrm{BH}$ for other HD patients.

Similarly, Da Silver et al. [15] performed a descriptive and exploratory study in Brazil to know the benefits of using the $\mathrm{BH}$ cannulation technique. They found that The $\mathrm{BH}$ technique has contributed satisfactorily and appreciably to the quality of life of patients. This was also one of the questions asked for satisfaction level in our study and only the BH group had higher satisfaction level on their quality of life compared to the RL group. According to the results of this study, patients using the $\mathrm{BH}$ cannulation technique reported a lower level of confidence using this technique than the RL group patients with a mean score of $3.41 \pm 1.06$ and $2.86 \pm 1.12$ for $\mathrm{BH}$ and $\mathrm{RL}$ respectively. We were not able to find similar studies that talked about the confidence level of patients using the $\mathrm{BH} /$ RL cannulation techniques. Maybe we were the first to conduct research on these cannulation techniques asking patients about their confidence level using these cannulation techniques.

In the "Satisfaction/confidence level for HD nurses who use the BH/RL technique" part of this study, we found that nurses who preferred using the $\mathrm{BH}$ cannulation reported low satisfaction-level score. While nurses who used the RL cannulation technique reported a high level of satisfaction, this was concluded after seeing the total mean score of $3.58 \pm 0.93$ and $2.50 \pm 0.81$ for $\mathrm{BH} / \mathrm{RL}$, respectively. Some similar studies also have been carried out to assess the satisfaction level of HD nurses who prefer the BH/RL technique. For instance, Struthers et al. conducted a randomized controlled trial in three dialysis centres to compare the $\mathrm{RL} / \mathrm{BH}$ techniques on patients and also involved HD nurses in 2010. Struthers et al.'s [9] study found that most of the HD nurses preferred the $\mathrm{BH}$ cannulation technique. The results of the study from 2010 are quite different from ours, because most the HD nurses in Nigeria preferred the RL and not the BH.

Nurses who prefer using the $\mathrm{BH}$ cannulation reported low confidence-level score, whereas nurses who use the RL cannulation technique reported a high level of confidence, with a total mean score of $3.92 \pm 0.72$ and $2.44 \pm 0.56$ for $\mathrm{BH} / \mathrm{RL}$, respectively. Some similar studies also have been carried out to know the confidence level of HD nurses who preferred the $\mathrm{BH} / \mathrm{RL}$ technique [10]. This study sought to find out if nurses are confident enough using the cannulation technique they prefer.

Ludlow [10] performed a prospective cohort study assessing the effects of cannulating the AVF using the $\mathrm{BH}$ technique from the perspectives of patients and nurses, using questionnaires rating confidence levels. High levels of staff confidence in $\mathrm{BH}$ technique were found. Ludlow's result [10] is contrasting with ours; our result showed that nurses who preferred RL were more confident.

\section{CONCLUSIONS}

Summing up the results, it can be concluded that the patients who are cannulated with the RL cannulation technique had a higher risk of experiencing complications than the patients using the BH site cannulation technique. It has been demonstrated that patients using the $\mathrm{BH}$ site cannulation technique had a higher satisfaction level than the RL patients, although the RL patients reported higher confidence levels than the BH patients. However, as for the nurses, the case is quite different. Because the use of the RL technique is common among the HD nurses in Nigeria, they tend to have higher satisfaction level and confidence level using the RL cannulation technique than the nurses who use the BH. The results suggest that this is the reason why the HD nurses using the $\mathrm{BH}$ technique have inadequate skills using the technique.

Complications from cannulation of an AVF fistula need to be avoided if at all possible by HD nurses. The risk of complications could be reduced with appropriate training of nurses, physicians, and patients. The HD nurses in Nigeria should be given adequate information for not using the RL technique in cannulating an AVF. The cannulation of a new mature access should be only performed by a skilled HD nurse to minimize the risk of cannulation-related problems. More skills on cannulation of AVF need to be acquired by the HD nurses in this study. Nurses who prefer using the RL cannulation technique need to be very strict with the antiseptic procedure to reduce AVF infection incidence among 
patients using the RL technique. Nurses should assess patients' pain tolerance before cannulation. Local anaesthetics could be used if needed especially for patients using the RL cannulation technique.

\section{ABBREVIATIONS}

$\begin{array}{ll}\text { AVF } & \text { : arteriovenous fistula } \\ \text { BH } & \text { : buttonhole } \\ \text { ESRD } & : \text { end-stage renal disease } \\ \text { ESRF } & : \text { end-stage renal failure } \\ \text { HD } & : \text { haemodialysis } \\ \text { KDOQI } & : \text { Kidney Disease Outcomes Quality Initiative } \\ \text { QOL } & : \text { quality of life } \\ \text { RL } & \text { : rope ladder } \\ \text { VA } & \text { : vascular access }\end{array}$

Acknowledgements: The authors would like to thank the medical director Dr. Ben Okpala in Rivon Clinic Port Harcourt, Nigeria, for the opportunity he gave to carry out this research work using the help of their patients from his haemodialysis unit. The authors gratefully acknowledge the help and participation of HD patient and HD nurses in this research work.

Authors' contribution: MJ involved in processing of the literature section, formulation of the research goal, object, hypotheses preparation and conducting of the survey, performing statistical analyses, making diagrams and tables, and writing of the manuscript. VR involved in counseling, preparation and conducting of the survey, making diagrams and tables, and writing of the manuscript. The authors read and approve the final version of the manuscript.

Ethical approval: The ethical approval for this study was obtained from Rivon Clinic in Port Harcourt, Rivers state Nigeria, after sending them the research aim, objectives, research questions, and the self-developed questionnaires for data selection, also an evidence form the Department of Nursing at Faculty of Health Sciences at Semmelweis University to show to the clinic in Nigeria that Jared Merit is a legal student in Semmelweis University. Informed consent was obtained from the patients and confidentiality was assured. No patients were forced to answer the questionnaire.

This manuscript was written based on Jared Merit's final thesis at the Faculty of Health Sciences at Semmelweis University, the theme leader was: Veronika Rajki, PhD, senior lecturer.

Conflicts of Interest/Funding: The authors declare no conflict of interest was received for this study. The publication of the article and the related research work did not receive any financial support.

\section{REFERENCES}

1. Malovrh M. Vascular access for haemodialysis: arteriovenous fistula. Ther Apher Dial. 2005;9(3):214-7.

2. McCann M, Einarsdottir H, Van Waeleghem JP, Murphy F, Sedgwick J. Vascular Access Management II: AVF/AVG cannulation techniques and complications. J Ren Care. 2009;35(2):90-8.

3. Bode AS, Tordoir JHM. Vascular access for haemodialysis therapy. In: Azar A, ed. Modelling and Control of Dialysis Systems. Studies in Computational Intelligence. Berlin/ Heidelberg: Springer; 2013. p. 269.

4. Schmidli J, Widmera MK, Basile C, et al. Editor's choice Vascular access: 2018 clinical practice guidelines of the European Society for Vascular Surgery (ESVS). Eur J Vasc Endovasc Surg. 2018;55(6) [cited 22 Feb 2019]. Available from: https://www.ejves.com/article/S1078-5884(18)30080-7/ fulltext

5. Hashmi A, Cheema MQ, Moss AH. Haemodialysis patients' experience with and attitudes toward the buttonhole technique for arteriovenous fistula. Clin Nephrol. 2010;74(5): 346-50.

6. Twardowski ZJ. Constant site (buttonhole) method of needle insertion for haemodialysis. Dial Transplant. 1995; 24(10):559-76.

7. Evans LM. Buttonhole cannulation for haemodialysis: a nursing review. RSAJ. 2012;8(3):146-51.

8. Verhallen AM, Kooistra MP, van Jaarsveld BC. Cannulating in haemodialysis: rope-ladder or buttonhole technique? Nephrol Dial Transplant. 2007;22(9):2601-4.

9. Struthers J, Allan A, Peel RK, Lambie SH. Buttonhole needling of ateriovenous fistulae: a randomized controlled trial. ASAIO J. 2010;56(4):319-22.

10. Ludlow V. Buttonhole cannulation in haemodialysis: improved outcomes and increased expense - is it worth it? CANNT J. 2010;20(1):29-37.

11. Smyth W, Hartig V, Manickam V. Outcomes of buttonhole and rope-ladder cannulation techniques in a tropical renal service. J Ren Care. 2013;39(3):157-65.

12. Parisotto MT, Schoder VU, Miriunis C, et al. Cannulation technique influences arteriovenous fistula and graft survival. Kidney Int. 2014;86(4):790-7.

13. Wong B, Muneer M, Wiebe N, et al. Buttonhole versus rope-ladder cannulation of arteriovenous fistulas for haemodialysis: a systematic review. Am J Kidney Dis. 2014;64(6): 918-36.

14. Chan MR, Shobande O, Vats H, et al. The effect of buttonhole cannulation vs. rope-ladder technique on haemodialysis access patency. Semin Dial. 2014;27(2):210-6.

15. Da Silva MD, Gurgel LJ, Escudeiro LC, Ferreira CH. Patient satisfaction with buttonhole technique. Cogitare Enferm. 2015;20(3):482-6.

16. Kacherova S. (2008, December 18). Vascular Access Assessment, Monitoring and Surveillance [WebEx session]. ESRD Network 18 [cited 2019 Aug 22]. Available from: http://www. presentica.com/ppt-presentation/vascular-access-assessmentmonitoring-and-surveillance-svetlana-lana-kacherova-esrdnetwork-18-qi-director-webex-s 